\title{
Selective Cytotoxicity of Counterion-Conjugated Charged Iron Oxide Nanoparticles: A Study with Lymphoblastoid Raji Cells
}

\author{
Goutam Ghosh $^{* 1}$, Archana Mukherjee ${ }^{2,5}$, Hari Narayan Bhilwade ${ }^{3,5}$, Alka Gupta ${ }^{4}$, Aruna Korde ${ }^{2,5}$, Rita \\ Mukhopadhyaya**4,5 \\ ${ }^{1}$ UGC-DAE Consortium for Scientific Research, Mumbai Centre, Trombay, Mumbai 400085, India. \\ ${ }^{2}$ Radiopharmaceuticals Division, Bhabha Atomic Research Centre, Trombay, Mumbai 400085, India. \\ ${ }^{3}$ Radiation Biology and Health Science Division, Bhabha Atomic Research Centre, Trombay, Mumbai 400085, India. \\ ${ }^{4}$ Molecular Biology Division, Bhabha Atomic Research Centre, Trombay, Mumbai 400085, India. \\ ${ }^{5}$ Homi Bhabha National Institute, Training School Complex, Anushakti Nagar, Mumbai 400094, India. \\ *E-mail: ghoshg@csr.res.in; ghoshg@yahoo.com; **E-mail: ritam@barc.gov. in
}

\begin{abstract}
The cytotoxicity of counterions-conjugated charged iron oxide nanoparticles (IONPs) was studied with lymphoblastoid Raji cells and was compared with peripheral blood lymphocytes. IONPs were coated either with tri-potassium citrate (TKC), or with cetylpyridinium chloride (CPC). TKC coated IONPs were negatively charged and $\mathrm{K}^{+}$counterions conjugated nanoparticles, and $\mathrm{CPC}$ coated IONPs were positively charged $\mathrm{Cl}^{-}$counterions conjugated nanoparticles. The cells were incubated with IONPs at $37{ }^{\circ} \mathrm{C}$ for $24 \mathrm{~h}$ and the cytotoxicity was studied by measuring the cell viability using MTT and LDH assays. The cytotoxicity of IONPs was further assessed through DNA fragmentation assay. Morphology of Raji cells was also observed by TEM. We have used a modified membrane lysis model to understand the cell death via cell membrane lysis due to counterions diffusion upon binding of IONPs.
\end{abstract}

Keywords Charged nanoparticles, Raji cells, blood lymphocytes, cell viability, cell membrane lysis.

\section{Introduction}

Research in nanomaterials opened up huge scope in various applications, especially in biomedical sciences [1-13]. Magnetic nanoparticles are ideal for accumulation in targeted tissues due to their host cell tropism, biophysical nature and low toxicity $[14,15]$. Nanomaterials modulate biological reactions such as inflammation and cell toxicity $[16,17]$. Iron oxide nanoparticles (IONPs) have been used in biomedical applications such as drug deliver and diagnosis. Interaction of surface modified IONPs with cell lines has been reported $[18,19]$. Reduced cell viability has been reported as the most common toxic effect of IONPS in in vitro studies. IONPs coated with different substances have shown different cell viability [20-23]. It has been thought that IONPs produce excessive ROS in the cell which cause DNA damage and lipid peroxidation [24].

There is no universal detection mechanism of cancer cells $[25,26]$, but a common feature such as net membrane charge may be useful. Metabolically active cancer cells, whether in vitro or in vivo, are known to secrete a large amount of anionic lactates [27] due to increased glycolysis causing the cell membranes to be net negatively charged [28]. Normal cell membrane, on the other hand, maintains charge neutrality (or, weakly positive charge) at the surfaces. Thus, charge modified nanoparticles can be targeted to cancer cell membrane for various theranostics applications and positively charged nanoparticles were reported to be more effective than negatively charged [29-32]. Once charged nanoparticles are bound to a cell membrane, subsequent interaction may affect the viability of the cell. Determination of the cell viability of cultured cells is essential prior to any cell based study such as cytotoxicity of chemicals. Cytotoxicity assays, such as MTT and LDH, are used to determine the cell viability in a culture after treating with chemical or pharmaceutical substance. Tetrazolium-based MTT assay used to determine cell viability whereas lactate dehydrogenase (LDH) assay is used for cell membrane integrity [24]. Shah et al. [33] studied the cell viability of HeLa cells after incubation with anticancer drug loaded and thermally responsive polymer coated superparamagnetic $\mathrm{MnFe}_{2} \mathrm{O}_{4}$ nanoparticles using MTT method and showed a huge reduction of cell viability upon release of the drug 
at $41{ }^{\circ} \mathrm{C}$. Using combined hyperthermia and drug therapy they proposed that composite nanoparticles would be effective agents of cancer therapy.

Earlier we reported irreversible unfolding of hen egg white lysozyme after incubation with oppositely charged counterions-conjugated IONPs. Electrostatic binding of proteins causes counterions to release from the charged coating of nanoparticles and diffuse into interior of bound proteins, disrupt their hydrophobic core and hydrogen bonds to unfold [34]. This model of unfolding of proteins was named the reverse charge parity counterions (RCPC) model [35] and has been observed to work successfully for different proteins [36, 37]. This model thus describes the selective toxicity of counterions-conjugated charged IONPs to proteins. It has also been reported that the increasing ionic radii of counterions such as $\mathrm{Li}^{+}<\mathrm{Na}^{+}<\mathrm{K}^{+}$in the coating of IONPs caused decreasing amount of unfolding of bound proteins [38]. Protein is a single molecular system which is an integral part of cell membrane. The cell membrane also contains bilayer of lipid molecules which are also composed of hydrophobic components and hydrogen bonds, like proteins. Therefore, in the present investigation, we chose to study the selectivity in toxicity of counterions-conjugated charged IONPs with cells as a higher order system, and lymphoblastoid Raji cells (cancerous) and peripheral blood lymphocytes (normal cells) were used for that purpose. The cells were incubated with IONPs at $37^{\circ} \mathrm{C}$ for $24 \mathrm{~h}$ and the cytotoxicity was studied by measuring the cell viability using MTT and LDH assays [24]. To our best knowledge this was not reported earlier.

\section{Materials and Methods}

\subsection{Materials}

$\mathrm{FeCl}_{3} \cdot 6 \mathrm{H}_{2} \mathrm{O}$ and $\mathrm{FeCl}_{2} \cdot 4 \mathrm{H}_{2} \mathrm{O}$ (both $98 \%$ pure) were purchased from Burgoyne Burbidges \& Co (India); $\mathrm{NH}_{4} \mathrm{OH}(30 \%)$ was purchased from Merck, India. Tri-potassium citrate (TKC, 98.5\%) was purchased from S. D. Fine Chem. Ltd. (India) and cetylpyridinium chloride (CPC, 98\%) was purchased from Sigma-Aldrich. TKC is an anionic citrate molecule $\left(\mathrm{C}_{6} \mathrm{H}_{5} \mathrm{O}_{7}^{-3}\right)$ conjugated with $3 \mathrm{~K}^{+}$ions, and $\mathrm{CPC}$ is a cationic cetylpyridinium molecule $\left(\mathrm{C}_{21} \mathrm{H}_{38} \mathrm{~N}^{-1}\right)$ conjugated with $1 \mathrm{Cl}^{-}$ion. All chemicals were used without further purification. Roswell park memorial institute (RPMI) medium, 10X Antibiotic solution, fetal bovine serum (FBS), 3-(4,5-dimethylthiazol-2-yl)-2,5- diphenyltetrazolium bromide (MTT), dimethyl sulfoxide (DMSO) and in-vitro toxicology assay kit: Lactic dehydrogenase (LDH) based were purchased from Sigma-Aldrich. Raji Cell line (Burkitt's lymphoma; B lymphocyte cell line) was procured from the National Centre for Cell Sciences, Pune. Suspension culture was maintained in RPMI medium supplemented with $10 \% \mathrm{FBS}$ at $37^{\circ} \mathrm{C}$, in humidified atmosphere containing $5 \% \mathrm{CO}_{2}$.

\subsection{Preparation of Charged IONPs}

Iron oxide nanoparticles (IONPs) were synthesized by chemical co-precipitation method under alkaline condition using the recipes reported earlier [34]. The black precipitate in reaction beaker was separated out using a strong magnet underneath and the supernatant was discarded. The black precipitate was washed several times by milli-Q water until the dispersion showed neutral $\mathrm{pH}$. Finally, after discarding the supernatant the precipitate was washed by acetone and kept for drying at room temperature.

For surface functionalization, $1.0 \mathrm{mg}$ of dried IONPs was added in each freshly prepared solution of $\mathrm{TKC}$ or $\mathrm{CPC}$ in milli-Q water, each at a concentration of $1.0 \mathrm{mg} / 1.0 \mathrm{~mL}$, and was put in ultrasonic vibration for $2 \mathrm{~h}$. In this process, we obtained black precipitate in each vial which was washed 2-3 times by milli-Q water (using a magnet underneath) to remove uncoated molecules of TKC or CPC from the IONPs dispersions [34]. The final dispersions were prepared by adding $1.0 \mathrm{~mL}$ of milli-Q water in each vial containing either TKC coated IONPs or CPC coated IONPs to make concentration of each dispersion as 0.1 wt.\%, w/v. The TKC and CPC coated IONPs were named IONP1 and IONP2, respectively.

\subsection{Culture and Passaging of Raji Cells}

Cell culture was carried out inside a clean room facility (Class 10,000) under laminar flow hoods (Class 
100). Raji cells were cultured in RPMI-1640 medium with 10\% FBS. Confluent cells were used for carrying out assays.

\subsection{Isolation of Lymphocytes from Whole Blood}

Human peripheral blood was diluted with RPMI-1640 medium (1:1) and carefully layered on Histopaque solution. Different layers were separated upon density gradient based centrifugation (using REMI, India) machine at $1500 \mathrm{rpm}(\mathrm{RCF} \sim 650 \times \mathrm{g})$ for $30 \mathrm{~min}$ at $20^{\circ} \mathrm{C}$ and the lymphocytes layers were collected in $15 \mathrm{~mL}$ sterile tube, washed at least 3 times by RPMI-1640 medium and used for experiments.

\subsection{Characterization Charged IONPs}

Size and zeta potential of charged IONPs in dispersions were measured at $25{ }^{\circ} \mathrm{C}$ using dynamic light scattering (DLS) (Zetasizer Nano ZS, Malvern, UK) technique with at least 3 runs for each sample obtain average final values and maximum error bars. The size of IONPs was also measured using a Libra 120 Plus transmission electron microscope (TEM, Carl Zeiss, Germany) operated at $120 \mathrm{kV}$. A drop of IONPs dispersion from each vial was put on carbon coated copper grids of 200 square meshes and left for drying at room temperature. At least 6 images were taken from different parts of both samples. The size distribution of IONPs was obtained using the Digital Micrograph software (Gatan Inc., USA) by counting 200-250 nanoparticles from different images for both the samples. The coating of IONPs was also examined using FTIR technique, as reported earlier [34].

\subsection{Cytotoxicity Assays}

For cytotoxicity assays, Raji cells $\left(\sim 0.5-1 \times 10^{4}\right.$ cells $\left./ 200 \mu \mathrm{L}\right)$ were plated in round bottom 96 well plate, $0.05,0.1,0.5$ and $1.0 \mu \mathrm{L}$ of $0.01 \mathrm{wt} . \%$ dispersions of IONP1 and IONP2 were added to cells in triplicates and incubated at $37^{\circ} \mathrm{C}$ for $24 \mathrm{~h}$. Then the plate was centrifuged at $450 \times \mathrm{g}(\mathrm{RCF})$ for 5 min in order to remove free nanoparticles from dispersions. The supernatant was examined by DLS and nanoparticles were detected. Precipitated cells were fed with fresh culture medium. Following the identical protocol blood lymphocytes were also incubated with IONP1 and IONP2, washed and redispersed in culture medium. The cell viability was investigated spectroscopically after carrying out MTT and LDH assays. Wells with only culture medium were used for background correction and cells without nanoparticles treatment as controls.

MTT is a colorimetric assay that spectroscopically measures the reduction of yellow MTT by mitochondrial succinate dehydrogenase to an insoluble, dark purple formazan which can occur only in metabolically active cells, i.e., MTT activity is a direct measure of cell viability. The formazan product is analyzed spectrophotometrically at $570 \mathrm{~nm}$ after dissolution in DMSO [39]. We added 50 $\mu \mathrm{L}$ of MTT solution $(5 \mathrm{mg} / \mathrm{mL})$ in each well containing either control or interacted cells in culture medium and incubated for $4 \mathrm{~h}$ in dark at $37^{\circ} \mathrm{C}$. Afterward, the plate was centrifuged at $450 \times \mathrm{g}$ for 5 min to precipitate cells and the supernatant containing the medium was discarded. Subsequently, 200 $\mu \mathrm{L}$ of DMSO was added followed by an addition of $25 \mu \mathrm{L}$ glycine buffer. The final dispersions of control (C.C.) and interacted cells (I.C.) were examined spectroscopically and the cell viability estimated using the following formulation:

$$
\text { Cell viability }(\%)=(\text { Absorbance of I. C. or C. C./Absorbance of C. C. }) \times 100
$$

$\mathrm{LDH}$, a soluble cytosolic enzyme, is released into culture medium when cells die via membrane fragmentation which is measured spectrophotometrically at $490 \mathrm{~nm}$ [40] and is proportional to the number of dead cells. We added $100 \mu \mathrm{L}$ of $\mathrm{LDH}$ assay mixture to each well containing either control (C.C.) or interacted cells (I.C.) in culture medium and the plate was covered with aluminium foil for incubation at room temperature for 20-30 min. Reaction was terminated by adding $15 \mu \mathrm{L}$ of $1.0 \mathrm{~N} \mathrm{HCl}$ to each well. Afterwards absorbance was measured at $490 \mathrm{~nm}$ and values were subtracted by absorbance at $690 \mathrm{~nm}$. The corresponding cell viability (\%) was estimated as (Absorbance of C.C./Absorbance of I.C. or C.C. $\times 100$. Both assays were repeated for at least 3 times with freshly thawed cells to check the reproducibility of results. 


\subsection{DNA Comet Assay}

For detection of DNA strand breaks in interacted cells, the alkaline comet assay (i.e., single-cell gel electrophoresis or SCGE) was performed [41]. Approximately $5 \times 10^{4}$ cells/well were incubated with $1.0 \mu \mathrm{L}$ dispersions $(0.01 \mathrm{wt} . \%)$ of IONP1 and IONP2 following the procedure mentioned above. Cells were centrifuged and pellets were mixed with $1.0 \mathrm{~mL}$ of $0.8 \%$ low melting agarose solution at $37^{\circ} \mathrm{C}$ making uniform layer on pre-chilled frosted slides. Two slides were made for each sample. Cell lysis was carried out overnight at $4^{\circ} \mathrm{C}$ in lysis solution $(2.5 \mathrm{M} \mathrm{NaCl}, 100 \mathrm{mM} \mathrm{Na} 2-\mathrm{EDTA}, 1 \%$ Triton X-100 and $10 \%$ DMSO, pH 13), slides were then washed with alkaline buffer and placed on a horizontal electrophoresis apparatus in alkaline buffer $(300 \mathrm{mM} \mathrm{NaOH}, 1.0 \mathrm{mM} \mathrm{Na2-EDTA,} \mathrm{pH} \mathrm{13)} \mathrm{at} \mathrm{room}$ temperature for 20 min to allow DNA unwind and expression of alkali labile sites. Electrophoresis was carried out in the same buffer at $0.6 \mathrm{~V} / \mathrm{cm}, 300 \mathrm{~mA}$ for $30 \mathrm{~min}$. Afterward slides were kept in neutralizing solution (0.4M Tris, $\mathrm{pH} 7.5)$ for 5 min to remove alkali and detergents. Staining was done using 1xSYBR green II dye and observed under a fluorescence microscope (Axioplane, Carl Zeiss, Germany) at 40X magnification. At random, 60 images per slide having IONP1 and IONP2 incubated Raji cells along with negative control were captured and evaluated using digital imaging software 'CASP'. Different parameters such as tail length (TL), and \%DNA in tail (\%DNA-T) were used for presenting DNA damage [41]. All data were expressed as mean \pm SE. Statistical analysis of data was carried out by one-way ANOVA using the Turkey-Kramer test. The differences were considered significant at $\mathrm{P}<0.05$.

\subsection{Morphological Study of Raji Cells: TEM}

Samples were prepared following a protocol described by Schrand et al. [42], using a step-by-step method for the mass-basis dosing of cultured Raji cells with IONPs, and the process of fixing, dehydrating, resin embedding, ultra microtome sectioning and subsequently visualizing the cell morphology by TEM. Samples were prepared for both control and, IONP1 and IONP2 incubated cells. For $24 \mathrm{~h}$ incubation at $37^{\circ} \mathrm{C}$, NP-dispersion and cells were taken in the ratio of $1 \mu \mathrm{L}: 10^{6}$ (vol/number). After incubation, IONPs were removed from growth medium by pelletizing the cells via centrifugation at $450 \times \mathrm{g}$ for $5 \mathrm{~min}$., and washed 3 times using phosphate buffered saline (PBS). Cells were fixed in $2.5 \%$ glutaraldehyde/formaldehyde in PBS at room temperature for $2 \mathrm{~h}$, washed 3 times with PBS. Following a post fixation with $1 \%$ osmium tetra oxide for $1 \mathrm{~h}$ and washing, the cells were immobilized in $1 \%$ low melting agar for further processing. Cells (in agar blocks) were dehydrated with graded series of ethanol $(30,60,75,90$, and $100 \%)$ for 15 min each. After removal of ethanol by treatment with propylene oxide, blocks were subsequently infiltrated with Spurr's resin and propylene oxide in ratios of 1:3, 1:1, and 3:1 (v/v) for $2 \mathrm{~h}$. Samples were then infiltrated with Spurr's resin for $16 \mathrm{~h}$ and embedded by incubation at $60^{\circ} \mathrm{C}$ for $72 \mathrm{~h}$. Resin blocks with embedded cells were then cut into $\sim 70 \mu \mathrm{m}$ thin sections using an ultramicrotome Leica EMUC7 (Leica, Germany) and placed on 200-mesh Formvar-coated copper grids for TEM.

\section{Results}

\subsection{Size of IONPs: TEM and DLS}

TEM image of IONP1 (Fig. 1(A)) indicated that the size distribution of nanoparticles was nearly uniform except few bigger agglomerations which have been removed by centrifugation. The histogram of uniform size distribution (inset) showed the mean around 7-8 nm. IONP2 also had similar image (not shown here). The size distribution measured by DLS for IONP1 (Fig. 1(B)) and for IONP2 (Fig. 1(C)) with mean diameter around 15 and $22 \mathrm{~nm}$ respectively, indicated agglomeration (due to weak van der Waals attraction) of $2-3$ IONPs in aqueous medium [34].

\subsection{Study of Surface Charge: $\zeta$-potential}

The surface charge of IONPs was examined via zeta $(\zeta)$ potential measurements (Fig. 2) which 
indicated that IONP1 $(-40 \mathrm{mV})$ had negative coating due to TKC and IONP2 $(+30 \mathrm{mV})$ had positive coating due to $\mathrm{CPC}$. High values of $\zeta$-potential implied that both IONPs were quite stable against bigger agglomeration. The specific surface coating of both IONPs was also confirmed by FTIR (not shown).

The $\zeta$-potential of Raji cell (in RPMI-1640 medium) was measured to be around $-15 \mathrm{mV}$ (Fig. 2) indicating that the cell membrane was rich with positive charge [27, 28].
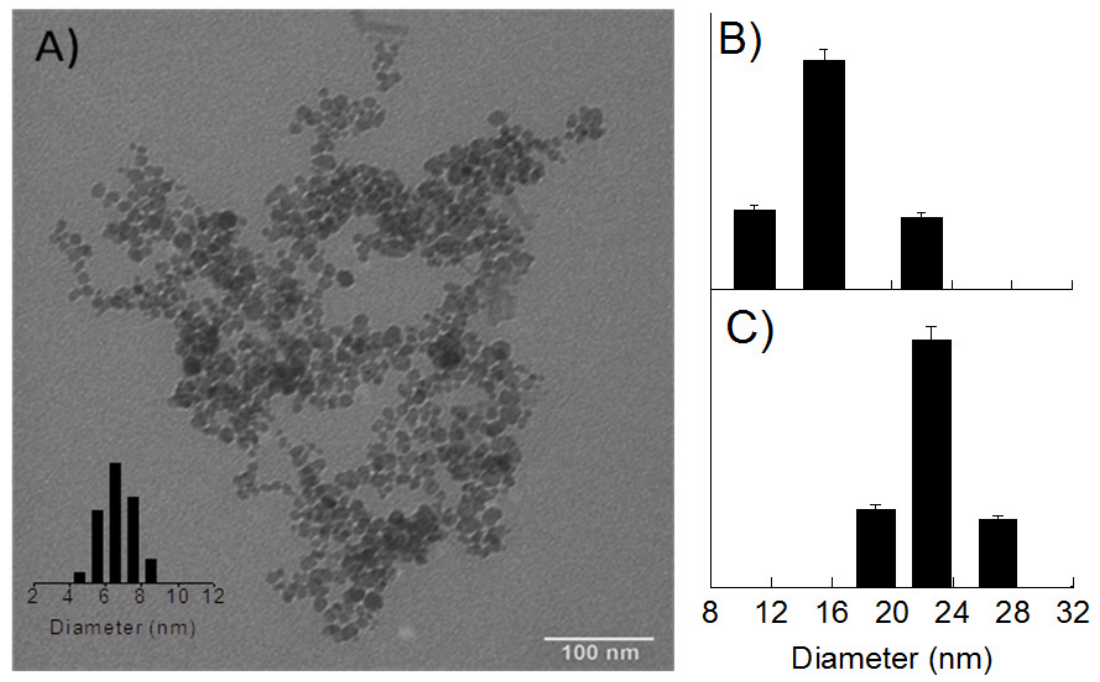

Figure 1. (A) TEM image of IONP1 and the histogram of size distribution (insets); size distribution of (B) IONP1 and $(\mathrm{C})$ IONP2 measured by DLS.

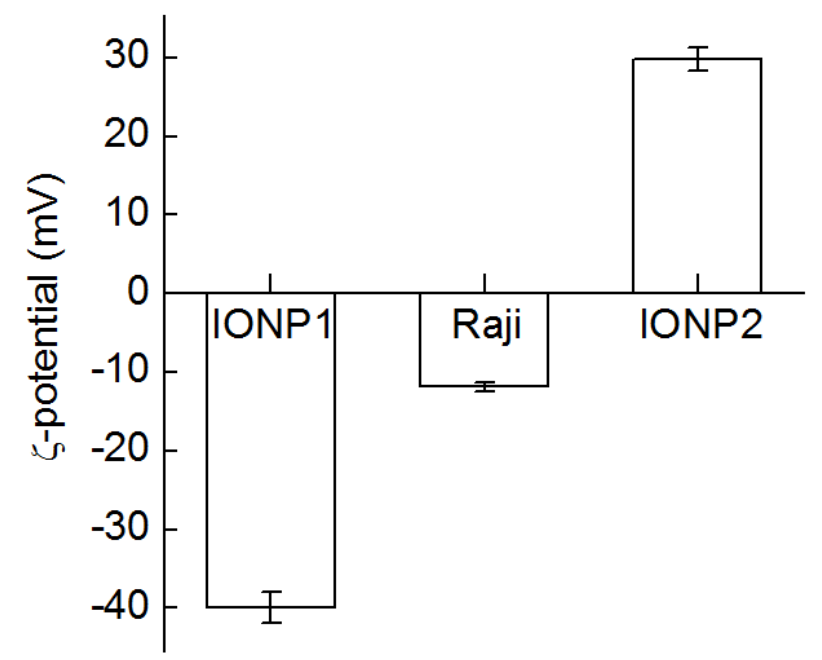

Figure 2. $\zeta$ - potential of IONP1, IONP2 and the Raji cell.

\subsection{Cytotoxicity: Raji Cells and Blood Lymphocytes}

Cytotoxicity of counterions-conjugated charged IONPs was investigated via cell viability study. The bar plots in Fig. 3 represented the cell viability (\%) of Raji cells, measured using MTT and LDH assays, at different dispersion volumes (i.e., IONPs concentrations) of IONP1 ((A) and (B)) and IONP2 ((C) and (D)). The error bars represented variations in the cell viability estimated from 3 set of measurements. Both assays provided fairly consistent results. Clearly, IONP1 had no systematic toxicity to Raji cells which may be due to repulsion of IONP1 by the cell membrane, as implied by the $\zeta$-potential data (Fig. 2). In contrast, IONP2 had systematic toxicity to the Raji cells which may be due to electrostatic 
binding of IONPs with cell membrane [43], as supported by the $\zeta$-potential data (Fig. 2). Optical images of Raji cells in control and those incubated with 1.0pL 0.01wt.\% IONP1 and IONP2, shown by the color plates at the side of Fig. 3, have also supported the assay results.

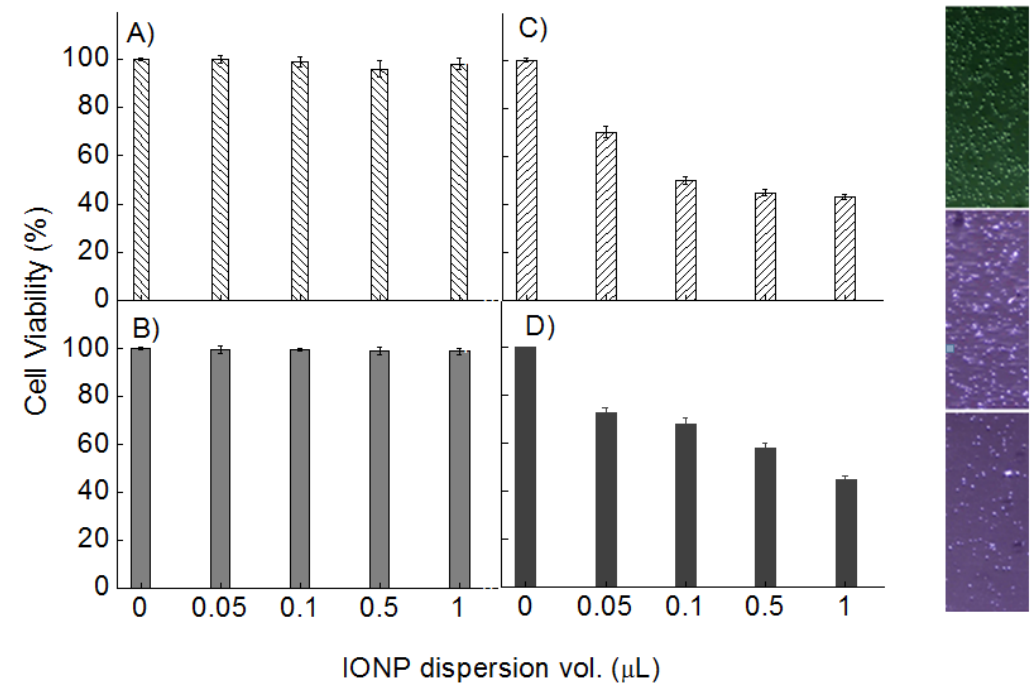

Figure 3. Cell viability (\%) of Raji cells, after incubation with IONP1: (A) MTT assay and (B) LDH assay, and with IONP2: (C) MTT assay and (D) LDH assay. Side coloured plates show optical images of Raji cells in control (top), and incubated with $1.0 \mu \mathrm{L} 0.01$ wt.\% dispersions of IONP1 (middle) and IONP2 (bottom).

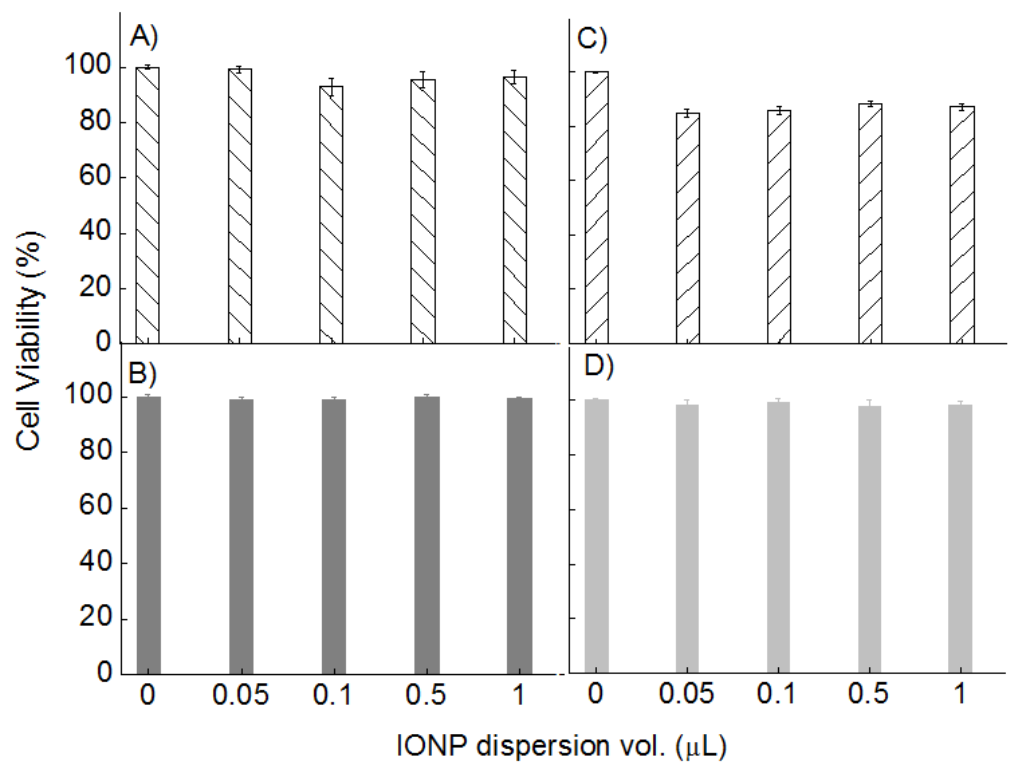

Figure 4. Cell viability (\%) of blood lymphocytes, after incubation with IONP1: (A) MTT assay and (B) LDH assay, and with IONP2: (C) MTT assay and (D) LDH assay.

The cell viability of peripheral blood lymphocytes measured using MTT and LDH assays (Fig. 4) showed no or very low toxicity of counterions conjugated charged IONPs. That implied a differential toxicity of counterions-conjugated charged IONPs for lymphoblastoid Raji cells (cancerous) and peripheral blood lymphocytes (normal cells). We have also not observed toxicity of TKC coated IONPs to Raji cells. Thus, our observation did not support the thought of producing ROS stress in living cells and cell damage by IONPs reported earlier [24]. Differential toxicity of IONPs to Raji cancer cells and blood lymphocytes was further supported by DNA comet assay study. 


\subsection{DNA Comet Assay: Raji Cells and Blood Lymphocytes}

DNA comet assay or single cell gel electrophoresis (SCGE) has been employed in an increasing number of studies directed toward genotoxic effects of environmental chemicals, radiation etc stress on human blood cells, fibroblasts and different cancer cell lines. We used this method in order to investigate association of nuclear damage with cell death after incubation with IONP1 and IONP2 at a fixed dose $(1.0 \mu \mathrm{L})$. Figure 5 (top plate) shows the images of single Raji cells for $(\mathrm{A})$ control, and those incubated with (B) IONP1 and (C) IONP2; and (D-F) show the software generated images of corresponding cells. Image of the control cell showed intact DNA, with small tail formation, whereas those incubated with IONPs showed significant DNA strand breaks with longer tail length (TL and \%DNA-T in Table 1). Cells incubated with IONP2 showed higher DNA damage and was in agreement with the cytotoxicity assay results.
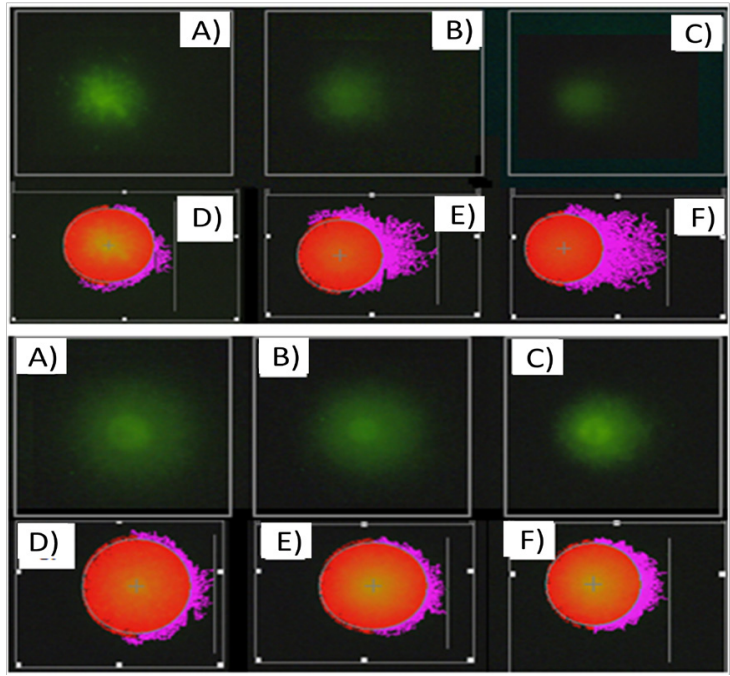

Figure 5. SCGE images of Raji cells (top plate) for (A) control, and incubated with (B) IONP1 and (C) IONP2. Software generated images of corresponding cells are shown in (D), (E) and (F), respectively. SCGE images of peripheral blood lymphocytes (bottom plate) for (A) control, and incubated with (B) IONP1 and (C) IONP2. (D-F) show software generated images of corresponding cells.

Table 1. Statistical parameters (\%DNA-T and TL) evaluated from SCGE assays for Raji cells in control and IONPs incubated dispersions.

\begin{tabular}{ccc}
\hline Raji cells & \%DNA-T & TL $(\mu \mathrm{m})$ \\
\hline Control & $9.68( \pm 0.47)$ & $63.49( \pm 2.56)$ \\
IONP1 incubated & $21.38( \pm 1.07)^{*}$ & $71.12( \pm 3.51)^{*}$ \\
IONP2 incubated & $26.07( \pm 1.93)^{*}$ & $87.92( \pm 5.03)^{*}$ \\
\hline
\end{tabular}

*values significantly higher than those of the control.

Figure 5 (bottom plate) shows the SCGE images of the blood lymphocytes for (A) control and those incubated with (B) IONP1 and (C) IONP2, and the corresponding software generated images are shown in $(\mathrm{D}-\mathrm{F})$. As observed DNA damage was nominal in lymphocytes after incubation with IONPs with small tail formation (Table 2).

Table 2. Statistical parameters (\%DNA-T and TL) evaluated from SCGE assays for peripheral blood lymphocytes in control and IONPs incubated dispersions.

\begin{tabular}{ccc}
\hline Lymphocytes & \%DNA-T & TL $(\mu \mathrm{m})$ \\
\hline Control & $5.17( \pm 0.88)$ & $26.85( \pm 6.09)$ \\
IONP1 incubated & $6.33( \pm 1.69)$ & $33.85( \pm 4.54)$ \\
IONP2 incubated & $8.02( \pm 0.60)$ & $38.92( \pm 4.67)$ \\
\hline
\end{tabular}




\subsection{Morphology of Raji Cells: TEM Study}

The morphology of Raji cells before (C.C.) and after (I.C.) incubated with counterions-conjugated charged IONPs was examined by TEM. Figure 6 shows images of Raji cells for (A) control, and incubated with (B) IONP1 and (C) IONP2. Cells of 10-15um with organelles like nucleus and mitochondria could be observed in all samples. Other organelles could not be observed, probably because sections were not contrasted with electron dense stains like uranyl acetate. Staining was avoided with the intent to observe nanoparticles, if any, in such cases and avoid false positives. The morphology of cells in control and those incubated with IONP1 appeared undistorted and dense, whereas those incubated with IONP2 appeared distorted and translucent. Enlarged images of distorted cells showed fragmented morphology (Figs. 6(D) and (E)). The fragmentation of the cell was probably occurred due to leakage of cell interior through fragmented cell membrane. Few localized nanoparticles (encircled by dotted line) were also observed in IONP2 incubated cells (Fig. 6(F)). Thus, the decay of the cell viability of Raji cells after incubation with IONP2 (Figs. 3(C) and (D)) was due to cell death via cell fragmentation. We used a modified cell membrane fragmentation model to understand the role of counterions in cell fragmentation.

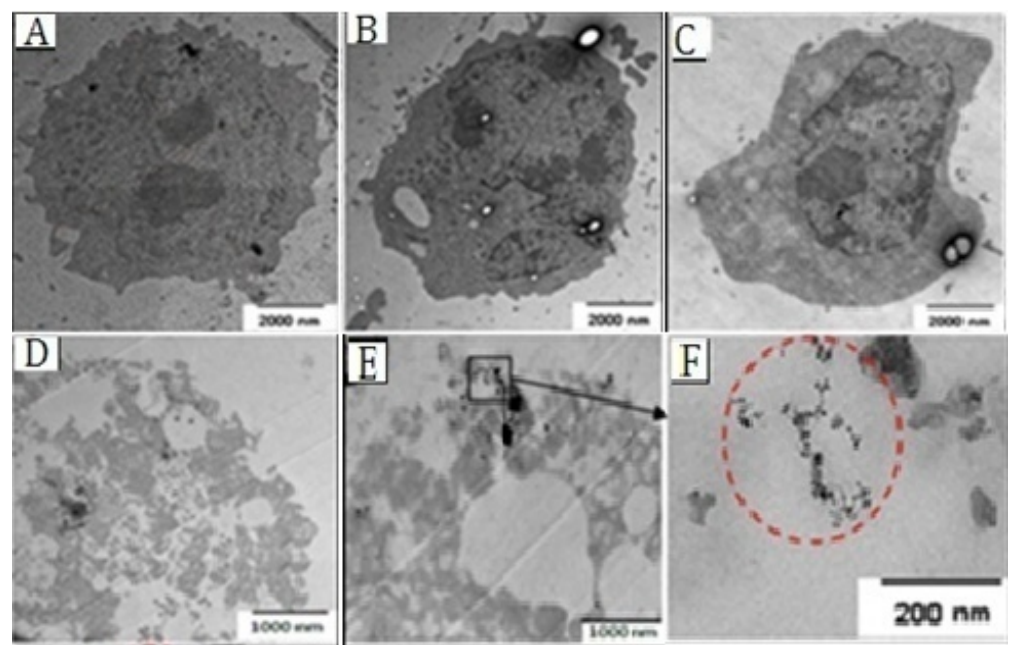

Figure 6. TEM images of Raji cells (A) before (control), and after incubation with (B) IONP1 and (C) IONP2. IONP2 incubated cell image was further magnified (D-E) to show fragmented morphology of the interacted Raji cells which also showed entrapped IONP encircled by red dashed circle in (F).

\section{Discussion}

Membrane rupture causes cell death as observed in mammalian cells [44-46]. Shillcock and Boal [47] developed a theoretical model on entropy driven rupture of a $2 \mathrm{D}$ cell membrane via formation of a circular hole. The model parameters were considered to be the membrane stress $(\sigma)$ and the edge tension $(\lambda)$ along the boundary of the hole, and the free energy barrier of the formation of hole was given by,

$$
\Delta F=F_{0}-F=-\sigma A+\lambda b n-k_{\mathrm{B}} T C n
$$

where $F_{0}$ and $F$ are membrane free energy before and after forming the hole. $A\left(=\pi R^{2}\right)$ is area of the membrane involving the hole of radius $R, b$ is a constant length and $n$ such length formed the boundary of the hole, $C$ is a constant and $k_{\mathrm{B}}$ is the Boltzmann constant. Values of the parameters used: $\sigma=$ $6 \times 10^{-3} \mathrm{~J} \cdot \mathrm{m}^{-2}$ and $\lambda=1-4 \times 10^{-11} \mathrm{~J} \cdot \mathrm{m}^{-1}[47]$.

Based on our experimental results, it was evident that only CPC coated IONPs were toxic to Raji cancer cells which could be realized using the RCPC model [35]: (i) electrostatic binding of CPC-IONPs with negative components of the cell membrane and (ii) subsequent release of counterions $\left(\mathrm{Cl}^{-}\right)$and unfolding of proteins or lipids in the membrane which caused fragmentation and cell death (Figs. 3(C) and (D)). The fragmentation of the cell membrane may be understood using the hole formation model explained above. In our case, the entropy $(S)$ per unit area of the membrane was considered to be a 
function of counterion distribution $(c)$, as $S=k_{\mathrm{B}} \ln c$. A rough estimation of the number of counterions per IONPs was carried out using the methods reported earlier [34]. Considering the number of cells in $1.0 \mathrm{~mL}$ of culture medium and number of IONPs in the added volume of nanoparticles for incubation an approximate number of IONPs, and in turn, approximate number $\left(c_{o}\right)$ of counterions per Raji cell was calculated. The counterion distribution $(c)$ in the cell membrane was reported to be Boltzmann type, $c=c_{o} \exp \left(-\frac{Z e \emptyset}{k_{\mathrm{B}} T}\right)[48]$ where $Z$ stands for valency of ion of charge $e\left(=1.6 \times 10^{-19}\right.$ coulomb), and $\emptyset$ represents membrane potential. Here, $Z e \emptyset$ represents electrical energy of single ion of valency $Z$. For $n$ ions, entropy was taken as $S=n k_{\mathrm{B}} \ln \left(c / c_{0}\right)=-n Z e \emptyset / T$. In our calculation, the free energy barrier of the formation of hole of radius $R$ was written as,

Substituting $S$, the free energy expression became

$$
\Delta F=-\pi R^{2}(\sigma+T S)+2 \pi R \lambda
$$

$$
\Delta F=-\pi R^{2}(\sigma-n Z e \emptyset)+2 \pi R \lambda
$$

Eq. 4 was independent of temperature $(T)$ and can be justified as the assays for control (C.C.) and interacted (I.C.) cells were carried out at same temperature $\left(25 \pm 0.1^{\circ} \mathrm{C}\right)$ and its relative effect in I.C. could be disregarded. For $\mathrm{Cl}^{-}$counterions $Z=1$, the membrane potential was taken as $-50 \mathrm{mV}$ [49], and $\sigma$ and $\lambda$ values were taken as above. The free energy of hole formation $(\Delta F)$ was calculated for different values of $n$ (indicating to different volumes of IONPs, i.e. 0, 0.05, 0.1, 0.5 and $1.0 \mu \mathrm{L}$ used in MTT/LDH assays) which as a function of $R$ (not shown) showed an energy barrier of the formation of hole. The barrier height was observed to decrease with increasing $n$ favoring the formation of bigger hole in the membrane. The \%ratio of $\Delta F$ 's obtained for I.C. to C.C. was considered as the cell viability (\%) and was compared with the experimental values in Fig. 7. Though there were differences in values but the trend of the calculated and the experimental cell viability as a function of IONP dispersion volume showed good agreement. Therefore, we may infer that the cell death observed from cytotoxicity assays and the cell fragmentation observed through TEM were coupled through membrane lysis due to intercalation of counterions upon binding of IONP2. In this regard, it is worth mentioning that there is no report on cell membrane lysis by CPC, though it has antibacterial activity [50].

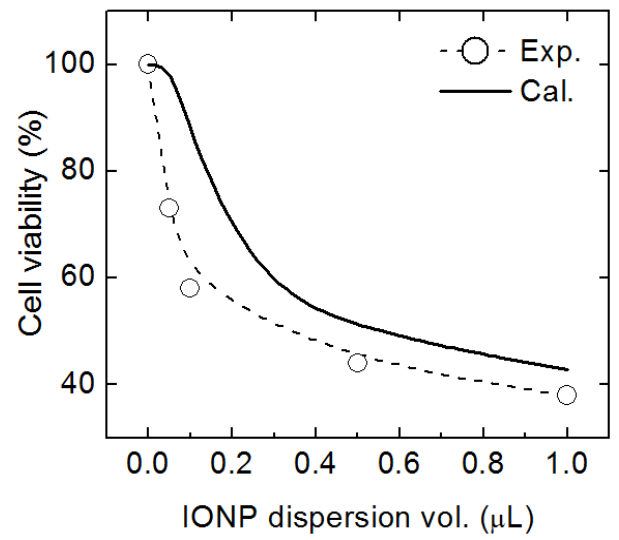

Figure 7. Cell viability (\%), versus ion number, plots of Raji cells obtained from experiments (-○- ) and theoretical calculation (-). The experimental points were obtained by averaging the cell viability (\%) derived from MTT and LDH assays at each concentration of IONPs. The ion number was calculated from the number of nanoparticles per cell at each concentration as mentioned in the text.

It is also to be noted that upon targeting the cell membrane (either by non-specific electrostatic binding [28] or by specific ligand-receptor binding) nanoparticles get internalized into cell via endocytosis mechanism [51], but there is no report so far on cell membrane lysis. Cancer cell membranes are characteristically different from normal cell membranes in many ways such as decrease in unsaturated and increase in saturated fatty acid contents which increases membrane fluidity [52], reducing membrane immunoglobulin ( $\mathrm{Ig}$ ) capping [53], changes in expression and functions of celladhesion molecules [54], carbohydrates alteration [28] and also over expression of negative charge [27]. As a result, strong electrostatic interaction takes place between the cancer cell membrane and foreign charged bodies like nanoparticles. This investigation suggested that the cytotoxicity of IONPs can be 
controlled and may be used for killing of cancer cells without affected much to the normal cells. Further investigations with different types of cancer cells may confirm these results and establish the therapeutic application of charged IONPs for cancer treatment.

\section{Conclusion}

We studied cytotoxicity of counterions-conjugated charged iron oxide nanoparticles (IONPs) to lymphoblastoid Raji cells and peripheral blood lymphocytes via MTT and LDH. It was observed that the positively charged IONPs with cetylpyridinium chloride (CPC) coating were significantly toxic to Raji cells in contrast to very low or no toxicity of tri-potassium citrate (TKC) coated negatively charged IONPs. Moreover, none of the IONPs showed systematic toxicity to peripheral blood lymphocytes. The differential observation between the cancer and normal cells was understood in terms of differences in their membrane charge. DNA comet assay also supported the observations made from the cytotoxicity assays. TEM study showed fragmentation of Raji cells affected by the incubation with CPC coated IONPs. The cell death and fragmented morphology of the cells have been explained using a modified cell membrane fragmentation model. This investigation suggested that the cytotoxicity of IONPs could be selective depending on the ions and charge on nanoparticles and may be used for killing of cancer cells without affecting much of surrounding normal cells. Further investigations with different cancer cells may confirm these results and establish the therapeutic application of charged IONPs for cancer treatment.

Acknowledgements. Authors acknowledge Dr. Lata Panicker, RB \& HSD, BARC, for giving access to DLS facility.

\section{References}

1. G. Oberdörster, "Safety assessment for nanotechnology and nanomedicine: Concepts of nanotoxicology", Journal of Internal Medicine, vol. 267, pp. 89-105, 2010.

2. C. E. Dreaden, A. M. Alkilany, X. Huang, C. J. Murphy, M. A. El-Sayed, "The golden age: gold nanoparticles for biomedicine", Chemical Society Reviews, vol. 41, pp. 2740-2779, 2012.

3. L. Dykman, N. Khlebtsov, "Gold nanoparticles in biomedical applications: recent advances and perspectives", Chemical Society Reviews, vol. 41, pp. 2256-2282, 2012.

4. F. Jia, X. Liu, L. Li, S. Mallapragada, B. Narasimhan, Q. Wang, "Multifunctional nanoparticles for targeted delivery of immune activating and cancer therapeutic agents", Journal of Controlled Release, vol. 172, pp. 10201034, 2013.

5. L. Mahmudin, E. Suharyadi, A. Bambang, S. Utomo, K. Abraha, "Optical properties of silver nanoparticles for surface plasmon resonance (SPR)-based biosensor applications", Journal of Modern Physics, vol. 6, pp. 10711076, 2015.

6. C. C. Berry, A. S. G. Curtis, "Functionalisation of magnetic nanoparticles for applications in biomedicine", Journal of Physics D: Applied Physics, vol. 36, pp. R198-R206, 2003.

7. Q. A. Pankhurst, J. Connolly, S. K. Jones, J. Dobson, "Applications of magnetic nanoparticles in biomedicine", Journal of Physics D: Applied Physics, vol. 36, pp. R167-R181, 2003.

8. S. Mornet, S. Vasseur, F. Grasset, E. Duguet, "Magnetic nanoparticle design for medical diagnosis and therapy", Journal of Materials Chemistry, vol. 14, pp. 2161-2175, 2004.

9. I. Safarik, M. Safarikova, "Magnetic techniques for the isolation and purification of proteins and peptides", Biomagnetic Research and Technology, vol. 2, pp. 7(1-17), 2004.

10. T. Neuberger, B. Schopf, H. Hofmann, M. Hofmann, B. von Rechenberg, "Superparamagnetic nanoparticles for biomedical applications: Possibilities and limitations of a new drug delivery system", Journal of Magnetism and Magnetic Materials, vol. 293, pp. 483-496, 2005.

11. S. Laurent, D. Forge, M. Port, A. Roch, C. Robic, L. V. Elst, R. N. Muller, "Magnetic iron oxide nanoparticles: Synthesis, stabilization, vectorization, physicochemical characterizations, and biological applications", Chemical Reviews, vol. 108, pp. 2064-2110, 2008. 
12. K. C. Barick, M. Aslam, Y. P. Lin, D. Bahadur, P. V. Prasad, "Novel and efficient MR active aqueous colloidal $\mathrm{Fe}_{3} \mathrm{O}_{4}$ nanoassemblies", Journal of Materials Chemistry, vol. 19, pp. 7023-7029, 2009.

13. C. Fang, M. Zhang, "Multifunctional magnetic nanoparticles for medical imaging applications", Journal of Materials Chemistry, vol. 19, pp. 6258-6266, 2009.

14. S. H. Moghimi, A. C. Hunter, J. C. Murray, "Long-circulating and target-specific nanoparticles: Theory to practice", Pharmacological Reviews, vol. 53, pp. 283-318, 2001.

15. J. W. M. Bulte, D. L. Kraitchman, "Iron oxide MR contrast agents for molecular and cellular imaging", $N M R$ Biomedicine, vol 17, pp. 484-499, 2004.

16. B. S. Zolnik, A. Gonzalez-Fernandez, N. Sadrieh, M. A. Dobrovolskaia, "Minireview: Nanoparticles and the immune system", Endocrinology, vol. 151, pp. 458-465, 2010.

17. H. Shi, R. Magaye, V. Castranova, J. Zhao, "Titanium dioxide nanoparticles: A review of current toxicological data", Part Fibre Toxicology, vol. 10, pp. 15(1-33), 2013.

18. F. Dilnawaz, A. Singh, C. Mohanty, S. K. Sahoo, "Dual drug loaded superparamagnetic iron oxide nanoparticles for targeted cancer therapy", Biomaterials, vol. 31, pp. 3694-3706, 2010.

19. L. Wang, H. Zhang, B. Chen, G. Xia, S. Wang, J. Cheng, Z. Shao, C. Gao, W. Bao, L. Tian, Y. Ren, P. Xu, X. Cai, R. Liu, X. Wang, "Effect of magnetic nanoparticles on apoptosis and cell cycle, induced by wogonin in Raji cells", International Journal of Nanomedicine, vol. 7, vol. 789-798, 2012.

20. S. Naqvi, M. Samim, M. Abdin, F. J. Ahmed, A. Maitra, C. Prashant, A. K. Dinda, "Concentration-dependent toxicity of iron oxide nanoparticles mediated by increased oxidative stress", International Journal of Nanomedicine, vol. 5, pp. 983-989, 2010.

21. E. Pawelczyk, A. S. Arbab, A. Chaudhry, A. Balakumaran, P. G. Robey, J. A. Frank, "In vitro model of bromodeoxyuridine or iron oxide nanoparticle uptake by activated macrophages from labeled stem cells: implications for cellular therapy", Stem cells, vol. 26, pp. 1366-1375, 2008.

22. Y. Ge, Y. Zhang, S. He, F. Nie, G. Teng, N. Gu, "Fluorescence modified chitosan-coated magnetic nanoparticles for high-efficient cellular imaging", Nanoscale Research Letter, vol. 4, pp. 287-295, 2009.

23. G. J. Delcroix, M. Jacquart, L. Lemaire, L. Sindji, F. Franconi, J. J. Le Jeune, C. N. Montero-Menei, "Mesenchymal and neural stem cells labeled with HEDP-coated SPIO nanoparticles: In vitro characterization and migration potential in rat brain", Brain Research, vol. 1255, pp. 18-31, 2009.

24. G. Liu, J. Gao, H. Ai, X. Chen, "Applications and potential toxicity of magnetic iron oxide nanoparticles", Small, vol. 9, pp. 1533-1545, 2013.

25. N. B. La Thangue, D. J. Kerr, "Predictive biomarkers: a paradigm shift towards personalized cancer medicine", Nature Reviews Clinical Oncology, vol. 8, pp. 587-596, 2011.

26. C. O. Madu, Y. Lu, "Novel diagnostic biomarkers for prostate cancer", Journal of Cancer, vol. 1, pp. 150-177, 2010.

27. D. C. Gadsby, "Ion channels versus ion pumps: the principal difference, in principle", Nature Reviews Molecular Cell Biology, vol. 10, pp. 344-352, 2009.

28. J. Chen, T. Liu, T. Gao, L. Gao, L. Zhou, M. Cai, Y. Shi, W. Xiong, J. Jiang, T. Tong, H. Wang, "Variation in carbohydrates between cancer and normal cell membranes revealed by super-resolution fluorescence imaging", Advanced Science, vol. 3, pp. 1600270(1-9), 2016.

29. L. Ghitescu, A. Fixman, "Surface-charge distribution on the endothelial-cell of liver sinusoids", Journal of Cell Biology, vol. 99, pp. 639-647, 1984.

30. A. L. Baldwin, N. Z. Wu, D. L. Stein, "Endothelial surface-charge of intestinal mucosal capillaries and its modulation by dextran", Microvascular Research, vol. 42, pp. 160-178, 1991.

31. A. C. Fleischer, C. K. Payne, "Nanoparticle-cell interactions: molecular structure of the protein corona and cellular outcomes", Accounts of Chemical Research, vol. 47, pp. 2651-2659, 2014

32. E. C. Cho, J. W. Xie, P. A. Wurm, Y. N. Xia, "Understanding the role of surface charges in cellular adsorption versus internalization by selectively removing gold nanoparticles on the cell surface with a $\mathrm{I}_{2} / \mathrm{KI}$ etchant", Nano Letter, vol. 9, pp. 1080-1084, 2009.

33. S. A. Shah, A. Majeed, M. A. Shafique, K. Rashid, A. U. Awan, "Cell viability study of thermo-responsive coreshell superparamagnetic nanoparticles for multimodal cancer therapy", Applied Nanoscience, vol. 4, pp. 227-232, 2014. 
34. G. Ghosh, L. Panicker, R. S. Ningthoujam, K. C. Barick, R. Tewari, "Counterion induced irreversible denaturation of hen egg white lysozyme upon electrostatic interaction with iron oxide nanoparticles: A predicted model", Colloids and Surfaces B: Biointerfaces, vol. 103, pp. 267-274, 2013.

35. G. Ghosh, "Counterion effects in protein nanoparticle electrostatic binding: a theoretical study", Colloids and Surfaces B: Biointerfaces, vol. 128, pp. 23-27, 2015.

36. G. Ghosh, L. Panicker, K. C. Barick, "Selective binding of proteins on functional nanoparticles via reverse charge parity model: an in vitro study", Materials Research Express, vol. 1, pp. 015017(1-12), 2014.

37. G. Ghosh, L. Panicker, "Interaction of human hemoglobin with charged ligand-functionalized iron oxide nanoparticles and effect of counterions", Journal of Nanoparticle Research, vol. 16, pp. 2800(1-10), 2014.

38. G. Ghosh, L. Panicker, K. C. Barick, "Protein nanoparticle electrostatic interaction: Size dependent counterions induced conformational change of hen egg white lysozyme", Colloids Surfaces B: Biointerfaces, vol. 118, pp. 1-6, 2014.

39. J. C. Stockert, A. Blázquez-Castro, M. Caŭete, R. W. Horobin, A. Lillanueva, "MTT assay for cell viability: Intracellular localization of the formazan product is in lipid droplets", Acta Histochemica, vol. 114, pp. 785-796, 2012.

40. F. K. M. Chan, K. Moriwaki, M. J. De Rosa, "Detection of necrosis by release of lactate dehydrogenase (LDH) activity", Methods in Molecular Biology, vol. 979, pp. 65-70, 2013.

41. H. N. Bhilwade, S. Jayakumar, R. C. Chaubey, "Age-dependent changes in spontaneous frequency of micronucleated erythrocytes in bone marrow and DNA damage in peripheral blood of Swiss mice", Mutation Research, vol. 770, pp. 80-84, 2014.

42. A. M. Schrand, J. J. Schlager, L. Dai, S. M. Hussain, "Preparation of cells for assessing ultrastructural localization of nanoparticles with transmission electron microscopy", Nature Protocol, vol. 5, pp. 744-757, 2010.

43. A. Chen, W. Le, Y. Wang, Z. Li, D. Wang, L. Ren, L. Lin, S. Cui, J. J. Hu, Y. Hu, P. Yang, R. C. Ewing, D. Shi, Z. Cui, "Targeting negative surface charges of cancer cells by multifunctional nanoprobes", Theranotics, vol. 6, pp. 1887-1898, 2016.

44. G. Kroemer, W. S. El-Deiry, P. Golstein, M. E. Peter, D. Vaux, P. Vandenabeele, B. Zhivotovsky, M. V. Blagosklonny, W. Malorni, R. A. Knight, M. Piacentini, S. Nagata, G. Melino, "Classification of cell death: recommendations of the nomenclature committee on cell death", Cell Death \& Differentiation, vol. 12, pp. 14631467, 2005.

45. P. Golstein, G. Kroemer, "Cell death by necrosis: towards a molecular definition", Trends in Biochemical Sciences, vol. 32, pp. 37-43, 2006.

46. W. Martinet, D. M. Schrijvers, G. R. de Mayer, "Necrotic cell death in atherosclerosis", Basic Research in Cardiology, vol. 106, pp. 749-760, 2011.

47. J. C. Shillcock, D. H. Boal, "Entropy-driven instability and rupture of fluid membranes", Biophysical Journal, vol. 71, pp. 317-326, 1996.

48. J. Wolfe, (2015) "Cellular thermodynamics: the molecular and macroscopic view" in eLS, Wiley, 2015, pp. 1-13.

49. A. A. Marino, D. M. Morris, M. A. Schwalke, I. G. Iliev, S. Rogers, "Electrical potential measurements in human breast cancer and benign lesions", Tumour Biology, vol. 15, pp. 147-152, 1994.

50. C. N. Cutter, W. J. Dorsa, A. Handie, S. Rodriguez-Morales, X. Zhou, P. J. Breen, C. M. Compadre, "Antimicrobial activity of cetylpyridinium chloride washes against pathogenic bacteria on beef surfaces", Journal of Food Protection, vol. 63, pp. 593-600, 2000.

51. S. Zhang, H. Gao, G. Bao, "Physical principles of nanoparticle cellular endocytosis", ACS Nano, vol. 9, pp. 8655-8671, 2015.

52. X. Meng, N. H. Riordan, H. D. Riordan, N. Mikirova, J. Jackson, M. J. Gonzalez, J. R. Miranda-Massari, E. Mora, W. Trinidad Castillo, "Cell membrane fatty acid composition differs between normal and malignant cell lines", Puerto Rico Health Sciences Journal, vol. 23, pp. 103-106, 2004.

53. H. J. Cohen, "Human lymphocyte surface immunoglobulin capping. Normal characteristics and anomalous behavior of chronic lymphocytic leukemia lymphocytes", The Journal of Clinical Investigation, vol. 55, pp. 8493, 1975 .

54. U. Cavallaro, G. Christofori, "Cell adhesion and signalling by cadherins and Ig-CAMs in cancer", Nature Reviews Cancer, vol. 4, pp. 118-132, 2004. 